\title{
Adhesive Characterization in Pre-Stressed Piezoelectric Laminates
}

\author{
C.A. Hodges ${ }^{\delta a}$, Karla Mossi ${ }^{\phi a}$ and Lisa Scott ${ }^{\xi b}$ \\ ${ }^{a}$ Virginia Commonwealth University, Richmond, VA 23284-3015 \\ ${ }^{b}$ Dominion Energy, Inc., Hampton, VA 23663-2206
}

\begin{abstract}
Pre-stressed piezoelectric laminates, consisting of one or more metal layers and a piezoelectric material bonded together with an adhesive, have been widely studied over the past few years, both numerically and experimentally. Most of the current research has concentrated on the effect of the metal layers, types and geometry, along with variations in the active layer of the laminate. Historically, the adhesive layer has been neglected as a contributing factor in the overall performance of the final device. This paper attempts to address the effect of the adhesive line thickness and its influence on the performance of pre-stressed piezoelectric laminates under specific boundary conditions. All laminates tested were constructed with the following lay-up: $0.354 \mathrm{~mm}$ thick stainless steel, adhesive, $0.381 \mathrm{~mm}$ PZT ceramic, adhesive, and a $0.0254 \mathrm{~mm}$ aluminum layer. The devices having an adhesive line thickness of $0.169 \mathrm{~mm}$ were classified as group A, and group B were the devices with an adhesive line thickness of $0.036 \mathrm{~mm}$. The adhesive line thickness for group A was approximately $21 \%$ more than the line thickness of group B. The devices were tested in a simply supported, free-free condition under a series of loads at a constant frequency of $5 \mathrm{~Hz}$ over a voltage range from 400 to 800 Volts peak-topeak. Displacement was measured using loads of 25, 50, 75, 100, and 200 grams for each actuator. The data from each group was averaged and compared. The results showed group B generated more displacement at the same "arm weight" applied as compared to group A. However, only three samples for group B were measured since the rest of the samples failed during testing. Failure of the devices of group B may be due to the ultimate stress of the devices and their ability to lift a load under those conditions. The study demonstrated that adhesive layer thickness, along with the manufacturing process, has to be taken into account when developing an application that requires load-bearing capabilities. Even though no direct mechanical property measurements were taken to verify this theory, the results demonstrated that the adhesive does play a critical role in the performance of the device as an actuator and should be factored into both experimental and numerical studies to obtain more accurate predictions of the ultimate behavior of these devices.
\end{abstract}

Keywords: Adhesive line thickness, pre-stressed piezoelectric actuators, loaded displacement

\section{INTRODUCTION}

Pre-stressed piezoelectric laminate actuators have been in existence for several years [1]. Applications however, are very scarce due to their high cost, manufacturability, and the limited data available regarding reliability, among other factors. Manufacturing and modeling of pre-stressed piezoelectric laminates with consistent performance and reliability is key to the development of any industrial or biomedical application. Depending on geometry, materials, and manufacturing processes, mechanical properties change and have a significant effect on the final product. Pre-stressed piezoelectric laminates are formed by bonding a ceramic piezoelectric layer to a conductive layer of material through the use of adhesives [2-7]. The adhesives vary from manufacturer to manufacturer, from the type of adhesive used to the amount (thickness) applied to the piezoelectric laminate devices. The importance of this aspect is sometimes overlooked. The

\footnotetext{
${ }^{\delta}$ Mechanical Engineering Undergraduate Student, Virginia Commonwealth University, 601 West Main Street, Room 314, P.O. Box 843015, Richmond, VA 23284-3015. Ph: (804) 828-9117, Fax:(804) 828-4269, E-mail: hodgesca@ vcu.edu

${ }^{\phi}$ Assistant Professor Mechanical Engineering, Virginia Commonwealth University, 601 West Main Street, Room 314, P.O. Box 843015, Richmond, VA 23284-3015. Ph: (804) 827- 5275, Fax:(804) 828-4269, E-mail: kmmossi@ vcu.edu

${ }^{\xi}$ Business Development Manager, Dominion Energy, Inc., NASA Langley Research Center, 6 West Taylor Street, Bldg. 1293C, Room 259, Hampton, VA 23681. Ph:(804) 864-4269, Fax: (757)-864-8312, E-mail: l.a.scott@larc.nasa.gov
} 
following results are presented from an investigation of the effect of the adhesive layer thickness on the mechanical properties of a pre-stressed piezoelectric laminates and its effect on the performance of the device as an actuator.

\section{EXPERIMENTAL SETUP}

The actuators tested consisted of strips of $12.7 \mathrm{~mm}$ wide by $76.2 \mathrm{~mm}$ long. The devices had several layers; stainless steel type 304, SI ${ }^{\mathrm{TM}}$ adhesive [8], PZT Type 3195HD ceramic (CTS Wireless), SI ${ }^{\mathrm{TM}}$ adhesive (Dominion Resources), and ASTM B20 aluminum foil; see Figure 1 for a schematic of this layout. The thickness corresponding to each of the laminate layers was $0.254 \mathrm{~mm}$ for the stainless steel, $0.381 \mathrm{~mm}$ PZT, $0.0254 \mathrm{~mm}$ for the aluminum, and varying thickness for the adhesive line. The devices were divided into two groups $\mathrm{A}$ and $\mathrm{B}$, with an average adhesive line thickness between each layer of $0.084 \mathrm{~mm}$ for group A, and $0.018 \mathrm{~mm}$ for group B. Group A consisted of 17 actuators and group B consisted of 6 actuators. Measuring three points along the length of the device using a Multitoyo caliper and averaging the results determined adhesive line thickness. Other measurements made included dome height shown in Figure 1, measured using a NAIS-LM10 non-contact laser; and capacitance, measured using a Fluke 189 multimeter. Furthermore, the devices were x-rayed for quality assurance prior to testing using a Philips MGC machine with film: Kodak Type M, Energy: 40 kvp +/- 5, current: 30 IDA, Time: 2 min, SFD: 26 inches.

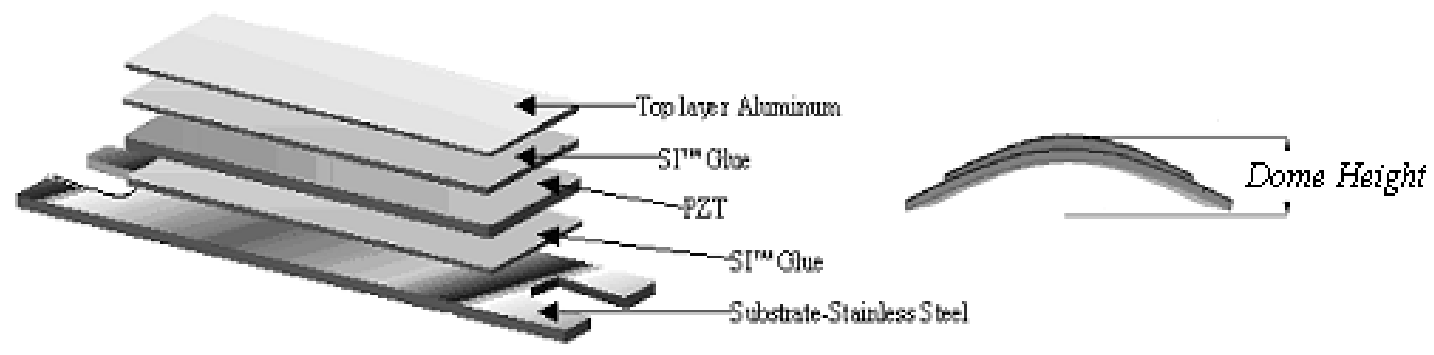

Figure 1 Actuator Layers

Displacement performance was evaluated under free-free boundary conditions at a constant frequency of $5 \mathrm{~Hz}$ with loads varied from 25 to 200 grams through a voltage range of $400 \mathrm{Vpp}$ to $850 \mathrm{Vpp}$. Data was acquired using a signal generator, an amplifier, an oscilloscope, a non-contact laser, and a data acquisition system. The signal generator was a Hewlett Packard 33120A 15MHz Function/Arbitary Waveform Generator that generated a signal of $5 \mathrm{~Hz}$ and a sine wave peakto-peak voltage of 2-4.25 Volts. The waveform generator voltage was amplified 200 times by a Trek Model PZD700 Dual Channel Piezo Driver, which increased the peak-to-peak voltage to 200-840 Volts. The displacement of the actuator was monitored with a NAIS LM10 (non-contact) Micro Laser Sensor. The actuators were placed under an "arm weight" of 25, 50, 75, 100, and 200 grams (free-free boundary conditions) concentrated at the center of the piezoelectric laminate device surface; see Figure 2 for a schematic of this setup. A LeCroy 9350L 500MHz Oscilloscope was used to display the data as it was collected by a PC through LabVIEW 6i. Hysteresis loop (displacement vs. voltage) comparisons were made between the piezoelectric actuators with the varying arm weights to determine the performance of each actuator and analyze the overall effect of the role of the adhesive on the performance of the actuator.

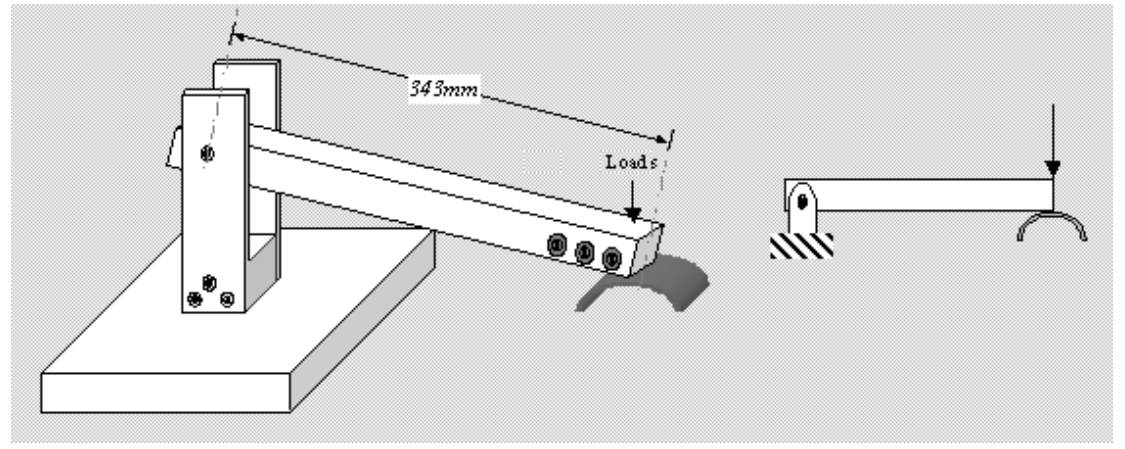

Figure 2 Arm Weight 


\section{RESULTS \& DISCUSSION}

Thickness and dome height measurements were taken to determine the mechanical properties of each group that may impact the performance of the device. These measurements are important since the values provide a measure of the stress distribution on the laminates; dome height also provides a parameter that is used in numerical modeling [9]. Average adhesive thickness was estimated for each group; group A was $0.084 \mathrm{~mm}$ thick, and group B $0.018 \mathrm{~mm}$ thick (Group B is 4.7 times thicker than group B), see Table 1 for a summary of these measurements. Dome heights and thicknesses were averaged for each group, and comparisons were made. The dome height for group B was found to be approximately $1.05 \%$ greater than group A, which is not a notable difference.

Table 1 Group A and B Basic Measurements

\begin{tabular}{|c|c|c|c|c|c|}
\hline & $\begin{array}{c}\text { Average Total } \\
\text { Thickness }\end{array}$ & $\begin{array}{c}\text { Adhesive line } \\
\text { thickness* }\end{array}$ & $\begin{array}{c}\text { Actuator } \\
\text { Weight }\end{array}$ & Capacitance & Dome Height \\
\hline Group & $(\mathrm{mm})$ & $(\mathrm{mm})$ & $($ grams $)$ & $(\mathrm{nF})$ & $(\mathrm{mm})$ \\
\hline Average "A" & 0.829 & 0.169 & 6.9 & 23.45 & 4.46 \\
\hline Standard Deviation "A" & 0.014 & 0.014 & 0.1 & 4.68 & 0.35 \\
\hline Average "B" & 0.697 & 0.036 & 6.4 & 31.47 & 4.68 \\
\hline Standard Deviation "B" & 0.006 & 0.006 & 0.2 & 2.29 & 0.21 \\
\hline
\end{tabular}

The critical data used to evaluate the displacement performance of the devices in each group were the loaded voltage displacement loops. A typical loop under a load has the shape indicated in the schematic in Figure 3. Note that a negative displacement means "downward" movement from the neutral position, and positive displacement means "upward" movement from the neutral position. Hence, positive displacement produces higher dome height, and negative displacement produces smaller dome height. A complete set of voltage-displacement loops is shown in Figure 4 for both groups, A and B, under loads of 25, 75 and 200 grams. It is important to notice that the loops for both groups, from top to bottom, that is, from lighter to heavier loads are "bowed" at the lower half of the voltage-displacement loop. This effect is larger as the load gets heavier, again for both groups. This trend suggests that the devices are deforming under the load making the loops larger at the bottom than at the top, since the top positive displacement correspond to lifting the load higher than the neutral position. At the lowest loads, 25 grams, the loop for group A is already "bowed," while group B is fairly uniform. However at the highest load measured, 200 grams, group A is less "deformed" than group B. This may be an indication of the pre-stress differences in each group, though pre-stress has not been measured directly.

To quantify this "bowed" effect a percent offset was calculated from the experimental data. The percent offset is defined as the difference between a straight line fit between the maximum and minimum displacement point. The maximum and minimum difference were then divided by the "span" of the loop, see Figure 3. These results indicate for group A that the offset at the highest voltage measured was $51 \%$ whereas group B exhibited offset of 57\%; which indicates that group B dissipates more energy than group A, making B most likely to fail under repeated loadings. These findings suggest this possibility, however, it is recommended that this observation be investigated further for a more detailed explanation.

Sample average displacement per load at different voltages for all the samples for group A is shown in Figure 5. The averaged results of each group showed that there was significant scatter on the displacement data, more than \pm one standard deviation. The results for group B on the other hand were not averaged because the final sample group consisted of three devices due to failures that occurred during testing in other devices. The failures may be due to microcracks inherent in the ceramic or formed during manufacturing and were not visible under x-ray pictures.

In order to better observe the positive and negative displacement, or the "upward" and "downward" movement of the device, the maximum and the minimum displacement at 50 grams and 200 grams for two pieces belonging to groups A and B, respectively, is shown in Figures 6. The graph illustrates that group A has less positive displacement than group B. This behavior has been documented recently by Ounaies et. Al [10] when the devices are subjected to high fields. 
This is probably due to the "bowing" effect of the loop, since maximum and minimum reading on the loops do not take into account the shape of the loop.

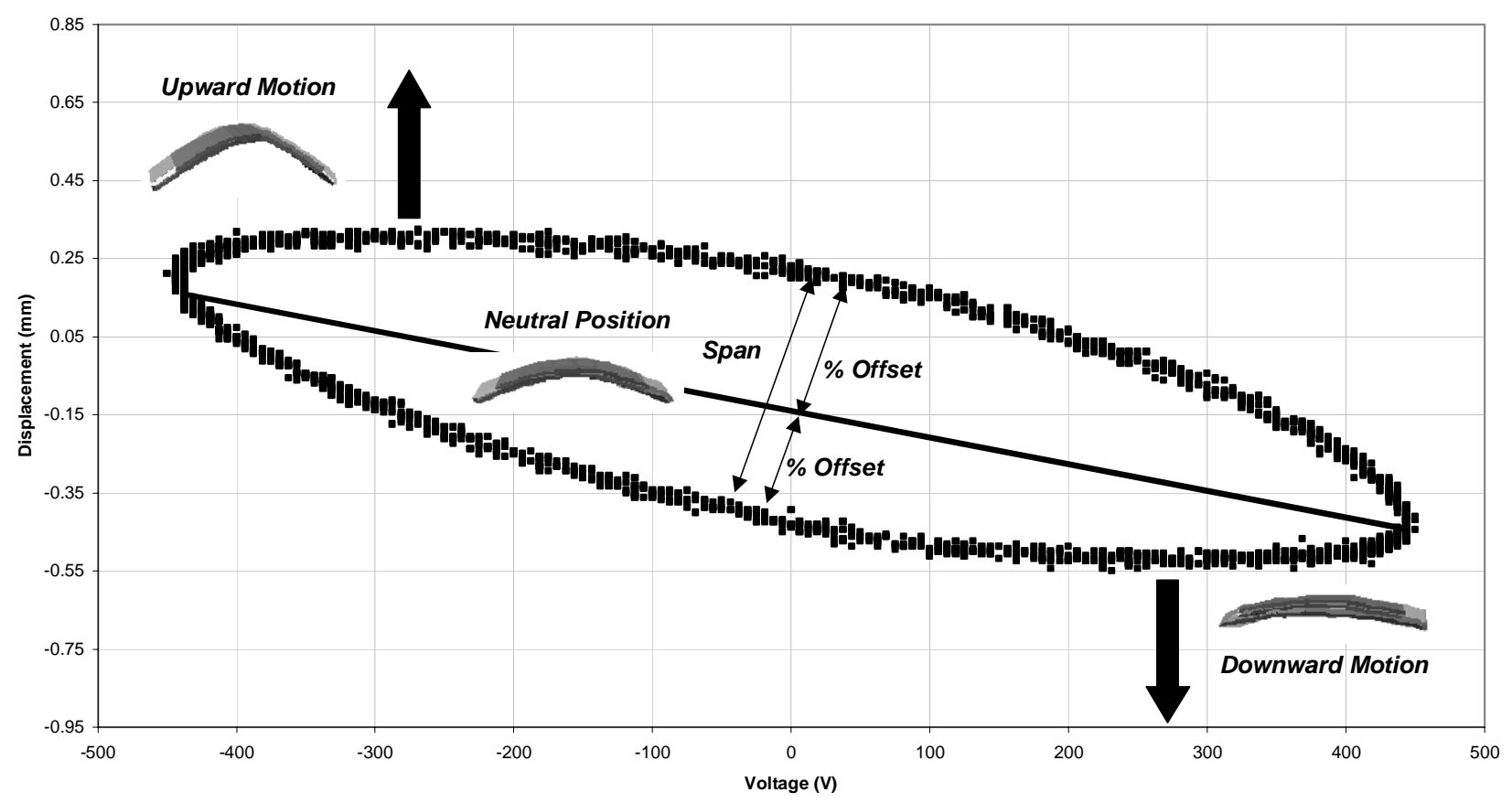

Figure 3 Schematic of the Loaded Displacement of a Typical Device

A more direct comparison of their performance can be made by looking at field applied vs. displacement, that is, voltage per unit thickness of the device, see Figure 7. This figure shows that at 50 grams load, group A and B have the same displacement when negative voltage is applied, contrary to when positive voltage is applied, group B shows a higher linear behavior. Note that the field applied is higher for group B, since the overall thickness is less for this group. This again is an indication of the total voltage applied to the thicker sample is smaller than the thinner one; therefore the thinner produces more displacement. In the same figure, group A and B at the heavier loads, 200 grams, show very similar performance when positive voltage is applied, which means downward movement. The inverse of this performance is observed at lower loads, that is same displacement for positive voltage applied but different for negative voltage applied. At intermediate loads, not shown in this figure, the total displacement, peak to peak, is very similar. For instance from Figure 4, for group A, with a 75 grams load and $850 \mathrm{~V}_{\mathrm{pp}}$, which is equivalent to $1025 \mathrm{~V} / \mathrm{mm}$ field shows a maximum and minimum displacement of +0.4 , and $-0.4 \mathrm{~mm}$. For group B, 75 grams, and $700 \mathrm{~V}_{\mathrm{pp}}$, or a field of $1004 \mathrm{~V} / \mathrm{mm}$ shows approximately the same values making the displacement of both groups equivalent. All of the above observations show that the ability to "lift" a load is better for group B. However, group B pieces seemed to have a lower ultimate stress value than group A (failure of most samples). In another words, B tends to fracture at the end of the elastic region, and A continues performing into the plastic region of the composite. 


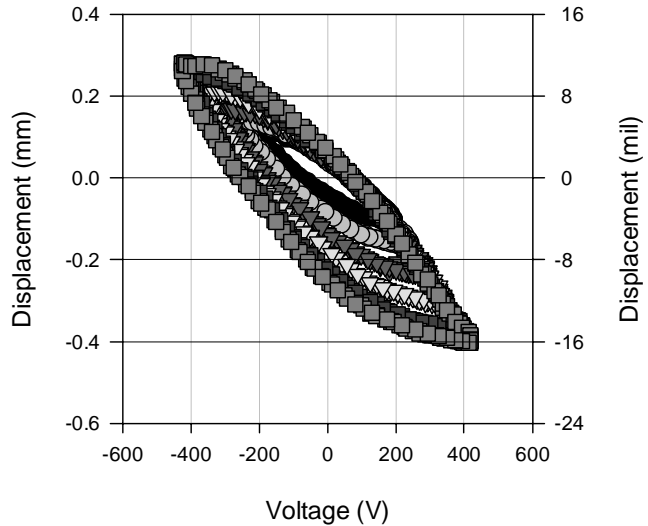

"A" 75 grams

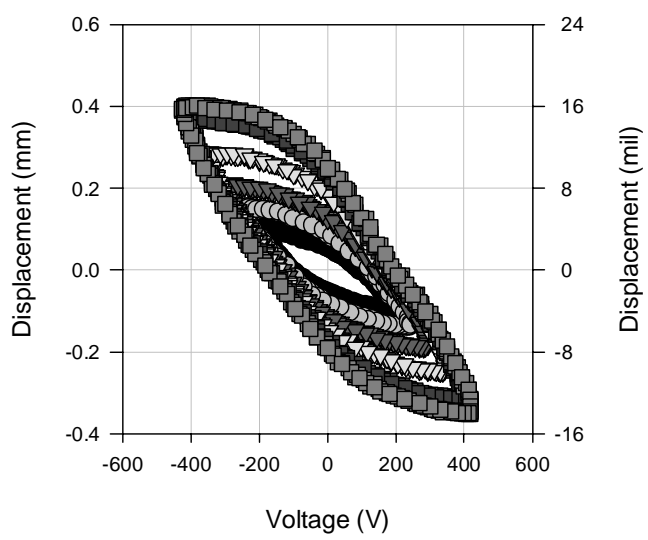

"A" 200 grams

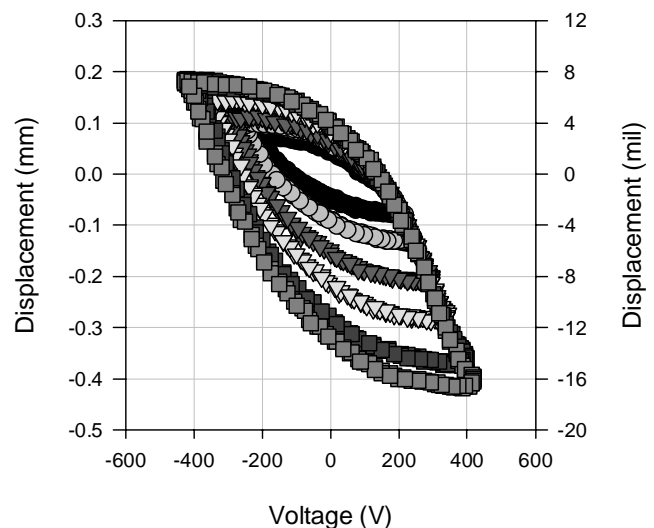

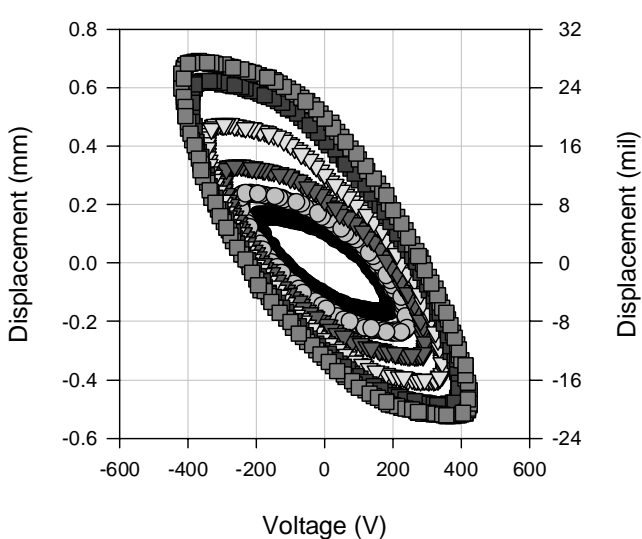

"B" 75 grams

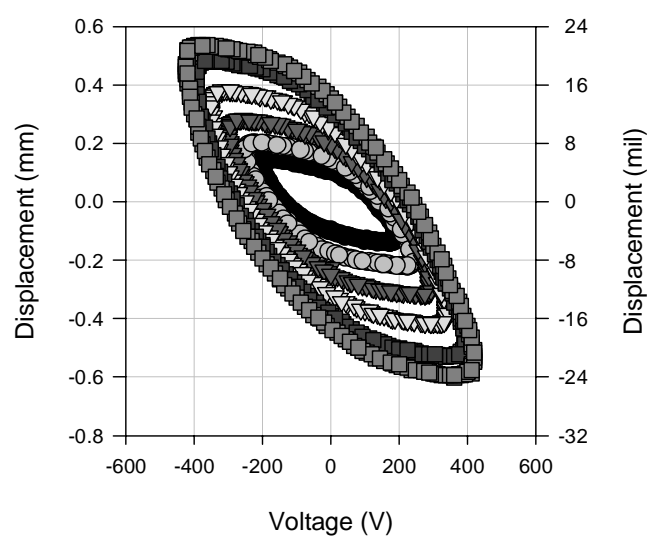

"B" 200 grams

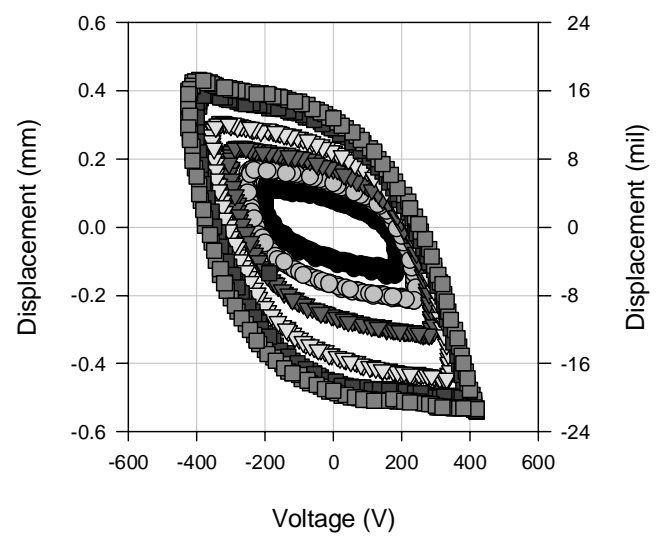

Figure 4 Typical Hysteresis Loop Comparison Groups A \& B 
"A" 400Vpp

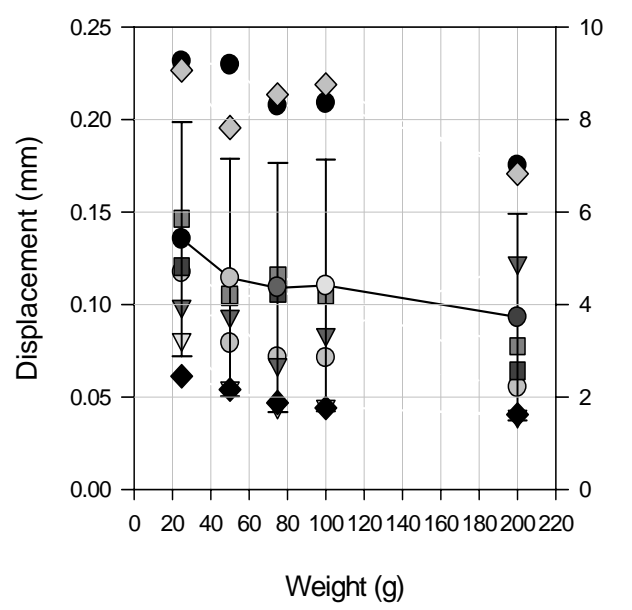

"A" 800Vpp

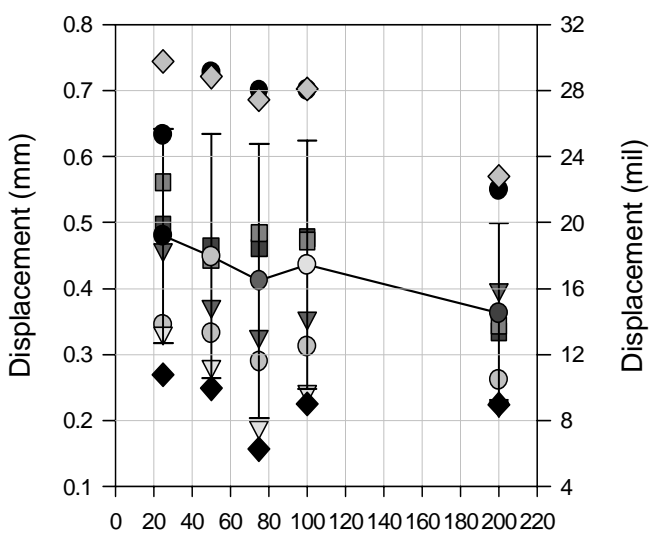

Weight $(\mathrm{g})$

Figure 5 Standard Deviation (peak-to-peak) Group "A"

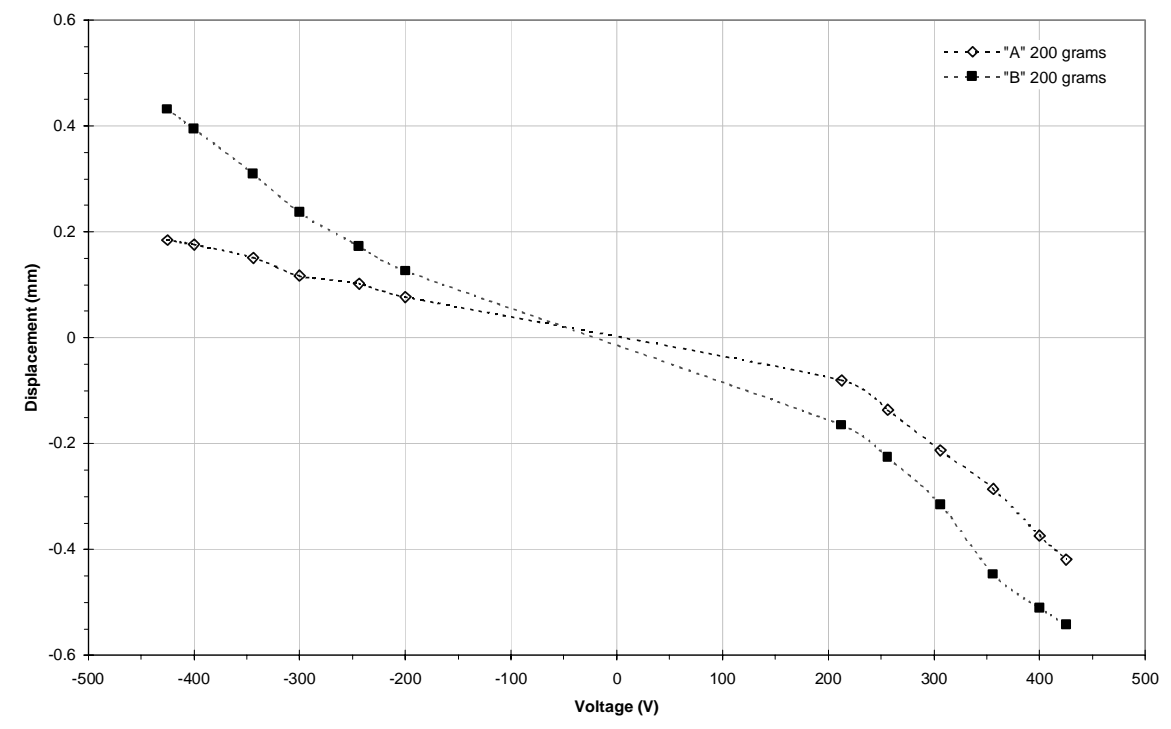

Figure 6 Voltage/Displacement Comparison (200 grams) Group A \& B

Another hypothesis is that there may be a substantial difference in the pre-stress of each group; potentially as a result of the difference in adhesive line thickness. Since pre-stress is the primary factor attributed to the enhanced performance of these devices over conventional piezoelectric laminates, determining the pre-stress associated with the device requires 


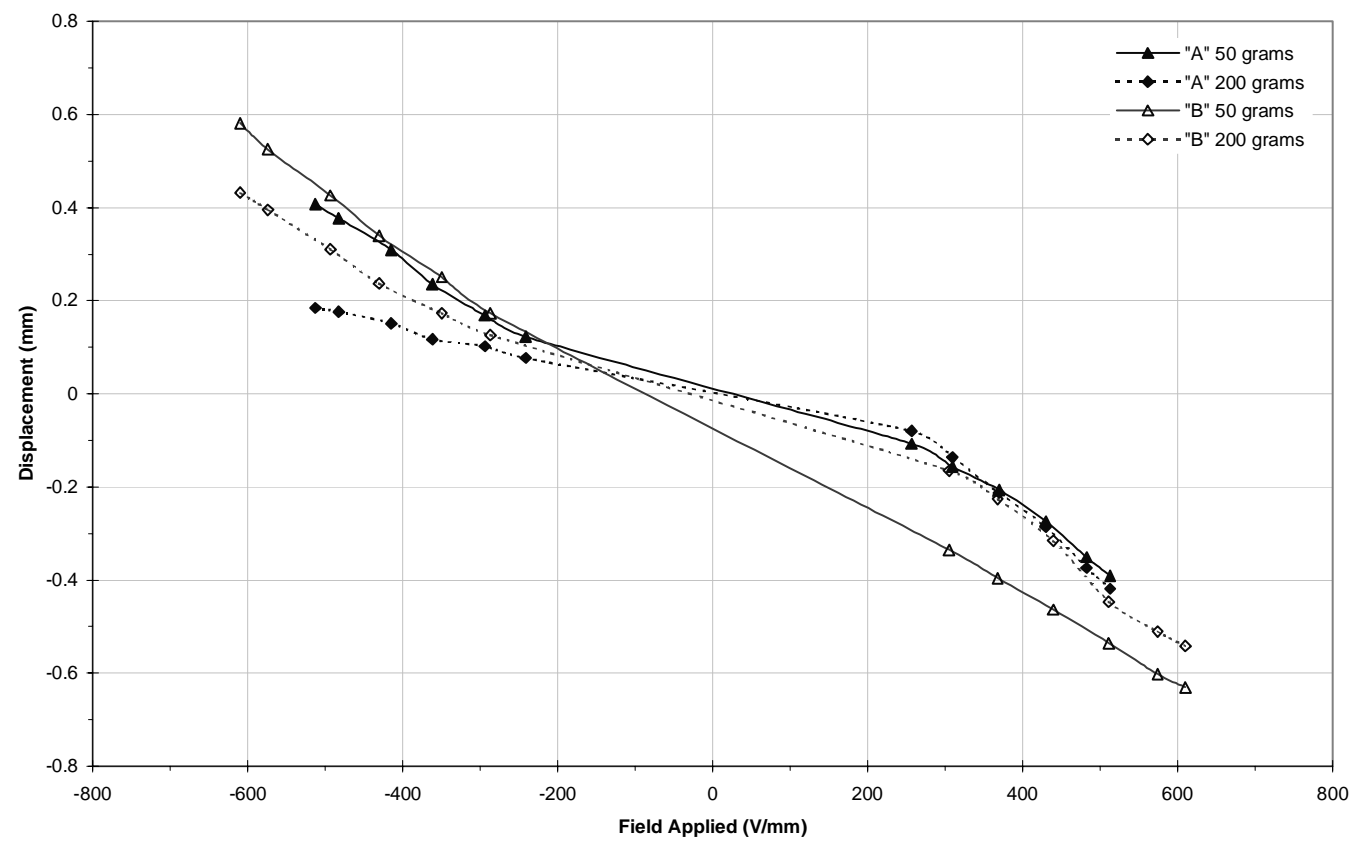

Figure 7 Field Applied/Displacement Comparison Group A \& B

extensive computational analysis. Since previous works have neglected incorporating this element in their model, it is difficult to clarify with certainty that the pre-stress is greatly affected by the adhesive line thickness. However, the results presented here indicate a strong possibility exists. The adhesive line thickness difference, while substantial between the two groups, does not appear to play a significant role in the overall stiffness of the device or the amount of pre-stress on the device (dome height). The adhesive line thickness however, plays a role on the ultimate stress of the device and its ability to lift a load. Other factors affecting the performance of the devices studied are the manufacturing techniques differences between the groups and may offer a plausible explanation as to why there is such a marked difference in the overall displacement performance.

\section{CONCLUSIONS AND RECOMMENDATIONS}

A study was conducted to determine performance effects of adhesive line thickness on pre-stressed piezoelectric laminate devices. Displacement measured under loads of 25, 50, 75, 100 and 200 grams, using a simply supported freefree boundary conditions system at different voltages, assessed performance. The devices were divided into two groups that had a markedly different adhesive line; group A had an adhesive line $21 \%$ thicker than group B. Group B generated more displacement at the same "arm weight" applied as compared to group A. However, only three samples for group B were measured since the rest of the samples failed during testing. Failure of the devices of group B may be due to the ultimate stress of the devices and their ability to lift a load under those conditions. No direct mechanical properties measurements were taken.

The results demonstrate that adhesive layer thickness, along with the manufacturing process, has to be taken into account when developing an application that requires load-bearing capabilities. Additional studies are needed to determine more accurately the mechanical properties of the composite and specifically the amount of pre-stress on the device when using the devices under a load. Furthermore, more direct methods to measure the adhesive layer thickness may be needed such as SEM. It is also recommended that future tests be performed on similar devices under identical conditions using comparable manufacturing processes. Life cycle tests are also suggested to determine if degradation occurs in the polymer as the device heats up for a thinner adhesive line thickness 


\section{REFERENCES}

[1] Lindner, Douglas K., DeGiorgi, Virginia G., McDennott, Sarah K., Integrated electronic/material/structural modeling of a smart material, SPIE 2000 Conference, 3041-02, March 1997.

[2] Mossi, K., Selby, G., Bryant, R. 1998. "Thin-Layer Composite Unimorph Ferroelectric Driver and Sensor Properties," Elsevier Science, Materials Letters 35 (1998) 39-49.

[3] Mulling, Usher, Dessent, Palmer, Franzon, Grant, and Kingon, "Load characterization of high displacement piezoelectric actuators with various end conditions". Sensors and Actuators Vol 94A, pp 19-24.

[4] K. Joon Yoon, Jae Han Chung, Nam Seo Goo, and Hoon. C. Park, Thermal Deformation and Residual Stress Analysis of Lightweight Piezo-composite Curved Actuator Device, SPIE 2001 Conference, 4333-68, March 2001. [5] Hoon Cheol Park, Sangki Lee, Byung Chan Cho, Kwang Joon Yoon, Nam Seo Goo, Three-dimensional assumed strain solid element for piezoelectric actuator/sensor analysis, SPIE 2001 Conference, 4326-58, March 2001.

[6] K. Mossi, Z. Ounaies, R. Smith, S.Oakley, "Geometrical Effects on Energy Production of a Thin Unimorph PreStressed Bender", To appear at the SPIE Conference, 4699-44, March 18-21,2002.

[7] K. M. Mossi and R. P. Bishop, "Characterization of Different types of High Performance THUNDER Actuators", Proc. of SPIE Conference, Newport Beach, CA, 1-5 March, Vol 3675-05, 1999.

[8] http://www.dom.com/productsinewtech/si-properties.jsp

[9] R. Wieman, R.C. Smith, T. Kackley, Z. Ounaies and J. Bernd, Displacement Models For Thunder Actuators Having General Loads and Boundary Conditions, CSRC Report, NC State.

[10]. Ounaies, Z., Mossi, K., Smith, R., and Bernd, J., "Low-field and high field characterization of THUNDER

actuators," ICASE Report No. 2001-9. 